\title{
Kernelized Correlation Filters Parameters Optimization for Enhanced Visual Tracking
}

\author{
Chor Keat Ong and Parvathy Rajendran \\ School of Aerospace Engineering, Universiti Sains Malaysia, Engineering Campus, \\ 14300 Nibong Tebal, Pulau Pinang, Malaysia
}

\begin{abstract}
Visual tracking has become one of the most important components in computer vision as the knowledge in this field can be applied into a wide range of applications in computer vision such as medical imaging, pattern recognition, video surveillance industrial robot, computer-human interaction, etc. A lot of researches have been conducted and many types of state-of-the-art methods and modifications such as sparse representation, online similarity learning, self-expressive, spatial kernel phase correlation filter and others are proposed in order to increase the robustness of the tracking. Despite of many methods has been demonstrated successfully but there are several issues that still need to be addressed. There still have some unsolvable difficulties in which they become a challenging task to track an object effectively and robustly and it will tend to decrease the accuracy of the results and hence. Until now, there are still no perfect algorithm to track the target flawlessly. In order to improve the performance, the main idea proposed is implementing optimization technique on the selected parameters and obtain a better performance. In this research, the tracking is proposed by using the Overlap Ratio (OR) and Centre Location Error (CLE). In our case, our target is to obtain a better accuracy which is higher OR and lower CLE than the result from the algorithms available in public. A simple optimization is used in here where the global best results with respect to the value of the parameters are selected through a range of values defined in our research. Through the optimization, the overall $\mathrm{OR}$ is increased to 0.554 and overall CLE is decreased to 19.803 pixels. Thus, the proposed method had increased the accuracy and robustness of the visual tracking on many of the video sequences.
\end{abstract}

$\underline{\text { Key words: Visual tracking, computer vision, kernelized correlation filters, parameter optimization, CLE, OR }}$

\section{INTRODUCTION}

Visual tracking is one of the research field in computer science that increases in popularity due to the importance to many applications in computer vision such as medical imaging, pattern recognition, video surveillance industrial robot, computer-human interaction, etc. Basically, the main objective of visual tracking is to estimate and locate the target objects in consecutive video frames.

In general, the basis working principle of visual tracking is after inputting a video sequence, we require a description for the object to be tracked. For example, shape, colour model, texture or others can use to be the template image of the object. Next, some context is applied into the object as implementing a good and proper integration of such context information into a tracking framework will bring some positive effects to visual tracking. After context information integration, the classifier classifies the image patches, then updated from time to time which is also known as online learning in order to handle and adapt the new appearance changes in the subsequent frames. These steps are repeated to track the object and stop when it reaches the last frame of the video.

Although, visual tracking has been studied for several decades but it is remaining as a challenging topic to be researched as mainly due to abrupt object motion, appearance pattern change, non-rigid object structures, occlusion and camera motion. And thus, there are no a single comprehensive method to handle all these destabilizing factors where these destabilizing factors are mainly consist of 11 attributes which are shown in Table 1 with description respectively (Wu et al., 2013; Yang et al., 2011).

In recent years, different algorithms have been proposed in order to solve the challenging issues. One of the methods is choose the right features or the most desirable property of a visual feature in order to be distinguished in the feature space easily. So, feature descriptors are playing an important role in selecting the

Corresponding Author: Parvathy Rajendran, School of Aerospace Engineering, Universiti Sains Malaysia, Engineering Campus, 14300 Nibong Tebal, Pulau Pinang, Malaysia 
Table 1: The 11 attributes annotated to test sequences with threshold values provided

\begin{tabular}{ll}
\hline Name & Description \\
\hline Illumination Variation (IV) & The illumination in the target region is significantly changed \\
Scale Variation (SV) & The ratio of the bounding boxes of the first frame and the current frame is out of the range $\left[1 / t_{\mathrm{s}}, \mathrm{t}_{\mathrm{s}}\right], \mathrm{t}_{\mathrm{s}}>1\left(\mathrm{t}_{\mathrm{s}}=2\right)$ \\
Occlusion (OCC) & The target is partially or fully occluded \\
Deformation (DEF) & Non-rigid object deformation \\
Motion Blur (MB) & The target region is blurred due to the motion of target or camera \\
Fast Motion (FM) & The motion of the ground truth is larger than tm pixels $\left(\mathrm{t}_{\mathrm{m}}=20\right)$ \\
In-Plane Rotation (IPR) & The target rotates in the image plane \\
Out-of-Plane Rotation (OPR) & The target rotates out of the image plane \\
Out-of-View (OV) & Some portion of the target leaves the view \\
Background Clutters (BC) & The background near the target has the similar color or texture as the target \\
Low Resolution (LR) & The number of pixels inside the ground-truth bounding box is less than $\left(\mathrm{t}_{\mathrm{s}}=400\right)$
\end{tabular}

right features. For instances, gradient feature is proved to have advantageous in human detection (Dalal and Triggs, 2005; Sabzmeydani and Mori, 2007); colour features which are robust against certain photomatic changes, texture features where texture is used to measure the intensity of a surface and quantifies properties such as smoothness and regularity (Fergus et al., 2003; Shotton et al., 2009; Winn et al., 2005), spatio-temporal features which used as representation for action recognition and visual detection, multiple features fusion which is more robust for image and video retrieval, visual tracking and detection.

Despite these feature descriptors, visual tracking still requires online learning based tracking methods to handle appearance variations of a target object. Online learning is required in for the tracker to adapt these appearance changes, adjust and update to new situations from time to time. There are 2 types of appearances variations which are intrinsic (pose changing, shape deformation) and extrinsic (occlusion, camera motion, camera viewpoint and illumination variation). Thus, these appearance variations must be handled by the online learning algorithm which is divided into 2 categories: generative method and discriminative method.

Appearance model: Generally, generative online learning method will learn the appearance of the object, then it will update online on the object model in order to adapt the appearance changes. Adam et al. (2006) represented the target using integral histogram and robust in target with partial occlusions or pose changes. Ross et al. (2008) presented an appearance-based tracker to gradually learn a low dimensional Eigen basis representation for tracking the target that with changing pose, illumination and appearance from time to time. Ross et al. (2008) Model is satisfying but it will encounter drifting problem. Jia et al. (2012) implemented a template update strategy which incremental subspace learning and sparse representation are combined together. The adaption of the template reduces possibility of drifting and the effect of the occluded target template.
Bao et al. (2012) proposed by adding an 12 norm regularization on the coefficients associated with the trivial templates into a new 11 norm related minimization model, it can achieved a better tracking accuracy than other 11 tracker, (Mei and Ling, 2009; Mei et al., 2011). Mei and Ling (2009) casted the tracking as a sparse approximation problem in a particle filter framework and achieved a very promising tracking result. Mei et al. (2011) presented a new approach known as Bounded Particle Resampling (BPR)-L1 tracker to enhance the template updates by detect occlusions and lessen the drifting problem.

Liu et al. (2013) proposed a new selection-based dictionary learning method known as $\mathrm{K}$-selection and modelled the target appearance by using a sparse coding histogram based on a learned dictionary. By this way, it can adapt to appearance changes and drifting problem is reduced. Liu et al. (2010) proposed a two stage sparse optimization to minimize the reconstruction error of the target and select a sparse set of features to maximize the discriminative power. Tian et al. (2015) gathered the sparse coefficients of all patches in an object into a histogram based on their spatial distribution. The candidates are predicted for object verification during tracking by using particle filter methodology. Sparse coding is implemented to evaluate degree of changes of the appearance model and thus reduced the drifting problem.

Cheng et al. (2015) had conducted both generative and discriminative trackers under the particle filter framework. Common method implemented by Cheng et al. (2015), Jia et al. (2012), Liu et al. (2010, 2013), Mei and Ling (2009) and Tian et al. (2015) is utilizing sparse representation to represent the target and their research prove that sparse representation is more powerful tool to handle and analysis appearance representation during online tracking where it had overcame many challenging attribute such as heavy occlusions, illumination changes and pose variation. Li et al. (2016a) embedded "Online Reconstruction Error Prediction (OREP)" into the IVT (Ross et al., 2008) framework to predict appearance 
reconstruction error and proven that OREP greatly improved the performance of some video sequences as compared with Bao et al. (2012) and Ross et al. (2008).

Meanwhile, discriminative learning method required a classifier to be trained and updated online to differentiate the object from the background. It is also known as tracking-by-detection because it requires the user to manually identify the target in the first frame to generate a set of features of target. Then, another separate set of features is generated automatically to describe the background. Next, the target will be separated from the background in the subsequent frames. Similarly, it must be updated continuously to handle the appearance changes.

Support vector tracking (Avidan, 2004) proposed SVM to optimize the classification score by generating a Gaussian pyramid from every support vector, known as "Support Vector Pyramid" to account large motions in the image plane. The experiment shows that it performs better in long period of vehicles tracking. Babenko et al. (2009) proposed online $\mathrm{MIL}$ algorithm for object tracking and achieves promising performance with real-time tracking. Henriques et al. (2012) proposed Fourier analysis that capable for extremely fast learning and detection with the fast fourier transform. Closed-form solutions for training and detection with several types of kernels including the popular Gaussian and polynomial kernels are derived and the algorithm achieved competitive performance.

Yang et al. (2014) proposed superpixels in an appearance model that gives flexible and effective mid-level cues to distinguish the background and the foreground target. This model is more capable to handle the situations with big changes of pose and scale, shape deformation, occlusion and camera shake. Zhang and Song (2013) presented online Weighted Multiple Instance Learning (WML) to integrate the sample importance into the learning procedure, compute a new bag probability function that combines the weighted instance probability. Patras and Hancock (2010) presented a discriminative framework that coupled the predictor to a probabilistic classifier to predict the target accurately.

Yuan et al. (2014) proposed a robust superpixel-based tracker via depth fusion, developed sufficient structural information and high flexibility of mid-level features, depth-map's discriminative ability for the target and background separation, thus generated stronger discriminative ability. Fan et al. (2014) presented a supervised approach to learn and update a structured, sparse and discriminative representation that alternating between robust sparse coding and dictionary updating. Zhuang et al. (2014) presented Discriminative Sparse
Similarity map (DSS map) to find the candidate with highest score in the evaluation model based upon a matrix and thus, obtain the best tracking results effectively.

Chen et al. (2016a) presented a robust Discriminative Local Collaborative (DLC). DLC encodes the candidates by an efficient local regularized least square solver with the 12 norm minimization by using the local image patches of both the target templates and the ones on the background cooperatively. Yang et al. (2016) applied Laplacian Regularized Least Squares (LapRLS) to learn a robust classifier for exploiting unlabelled data and preserving the local geometrical structure of the feature space adequately.

Qian and Xu (2016) presented Subclass Discriminant Constraint (SDC) for visual tracking. The SDC searches for a discriminative subspace to allow linear separation of image blocks that connected with the object and the background. Two dictionaries are constructed and learned in such subspaces for tracking and detection. A transformation matrix and sparse coefficient codes are being found out during dictionary learning. The similarity between the target candidate and the template is determined over sparse coefficients according to the histogram intersection.

Correlation filter: Correlation filter based tracking (Bolme et al., 2010) utilized filters trained on example images to model the appearance of objects. The object is initially selected by a tracking window that centred on the object in the first frame. By correlating the filter over a search window in next frame tracking and filter training collaborate to track the target. Next the new position of the target is indicated from the location respective to the maximum value in the correlation output. Based on this new location, appearance variation is updated online. Fourier domain Fast Fourier Transform (FFT) is applied to compute correlation to generate a fast tracker.

Zhang et al. (2016) presented spatial kernel phase correlation based tracker (SPC) that only implements phase correlation filter on adoption of the phase spectrum to estimate the object's translation. SPC achieves favourable tracking performance as it is more robust to noise and cluster. Liu et al. (2016b) presented a partbased representation tracker via kernelized correlation filter for visual tracking and Spatial-Temporal Angle Matrix (STAM) that used to select reliable patches from parts via multiple correlation filters to obtain stable patches effectively. Combination of this framework increases the diversity of affine matrices and related candidates.

Chen et al. (2016b) proposed a patch based tracker which adaptively integrates the kernel correlation filters 
with multiple effective features to handle occlusion challenges. The effective patches are selected by using an adaptive weight selection scheme and thus, improves the robustness of algorithm. Li et al. $(2016 \mathrm{a}, \mathrm{b})$ presented a multi-view correlation tracker where multi-view model fuses various features and more discriminative features is selected. Fast training and efficient target locating provided by correlation filter framework had enhanced stability of scale variation tracking.

Others state of art method: Sevilla-Lara and LearnedMiller (2012) proposed Distribution Fields (DFs) to build an image that allows smoothing the objective function and the information about pixel values is keep intact. DFs descriptor has the advantage on slow changes in appearance and pose and minor occlusions. Zhang et al. (2012) proposed Compressive Tracking (CT) to preserve the structure of original image space based on non-adaptive random projections. By adopting a very sparse measurement matrix, features from the foreground and background targets are compressed efficiently. Generative and discriminative appearance models are combined in CT algorithm to encounter for scene variations.

Grabner et al. (2006) presented an on-line AdaBoost feature selection algorithm that has an advantages on its capability of on-line training, allowing the adaption of the classifier while tracking the object. Thereby appearance changes of the object such as out of plane rotations, illumination changes are handled effectively. Oron et al. (2015) proposed Locally Orderless Tracking (LOT) that will estimate the amount of local (dis) order in the target automatically, allows the tracker specific in both rigid and deformable objects on-line without prior assumptions.

Dinh et al. (2011) proposed auto exploration on the context information in two semantic terms which are distracters and supporters by using a sequential randomized forest an online template-based appearance model and local features. The tracker able to handle some challenges in tracking in uncontrolled environments with abrupt motion, occlusion, motion blur and frame-cut. Grabner et al. (2008) proposed a novel on-line semi-supervised boosting method to reduce drifting problem in tracking applications. The update process is formulated in a semi-supervised fashion as combined decision of a given prior and an on-line classier without adjusting any parameters.

Kwon and Lee (2010) proposed visual tracking decomposition scheme that efficiently highlights the object with drastic changes of motion and appearance or both. Zhuang et al. (2016) proposed a shallow and deep collaborative model that collaborates generative model to construct a local binary mask for handling occlusion tracking and a discriminative classifier to learn generic features. Cooperation between of these two models is more favourable to overcome occlusion and target appearance change.

Hu et al. (2016) proposed a Deep Metric Learning (DML) approach for under the particle filter framework that utilizes a feed-forward neural network architecture to classify the target object and background regions. A set of hierarchical nonlinear transformations in the feed-forward neural network is learned in order to project both the template and particles into the same feature space. The marginal between objects and backgrounds are maximized and thus, that objects are separated from the background regions easily.

\section{MATERIALS AND METHODS}

In this study, we will discuss on the basic concept of high speed tracking with Kernelized Correlation Filters (KCF) (Henriques et al. 2015). The parameters and idea of optimization technique used to optimize the performance of KCF in our research is presented with initial setting and procedures required. The research flow is presented in a flow chart to highlight the crucial procedures in our framework. The proposed method will be evaluated by its tacking performances which is OR and CLE where the calculation for both evaluator is discussed in study below.

Proposed method: The proposed method is basically a simple modification on the KCF. Instead of the default values, two parameters are selected to be varied in the optimization process which are $\mathrm{p}$ (padding) and $\mathrm{s}$ (spatial bandwidth). Padding is the extra area surrounding the target while spatial bandwidth is used to predict the response of an imaging system to very small objects which directly related to the size of the image and its object.

The range of values are defined at first in order to determine the global best values that are corresponding to the best result obtained. At first, the original setting for padding and spatial bandwidth is 1.5 and 0.1 , respectively. By altering the values of padding from 1-2 with increment of 0.1 while $0.05-0.5$ with increment of 0.05 for spatial bandwidth, the OR and CLE will be computed at each combination. Then, the global best tracking performance is obtained from all the combinations and recorded for all different sequences.

However, for some video sequences, the results obtained cannot achieved any improvement within the combination of these values. Thus, we increase the range of values to be optimized where padding is increased from 


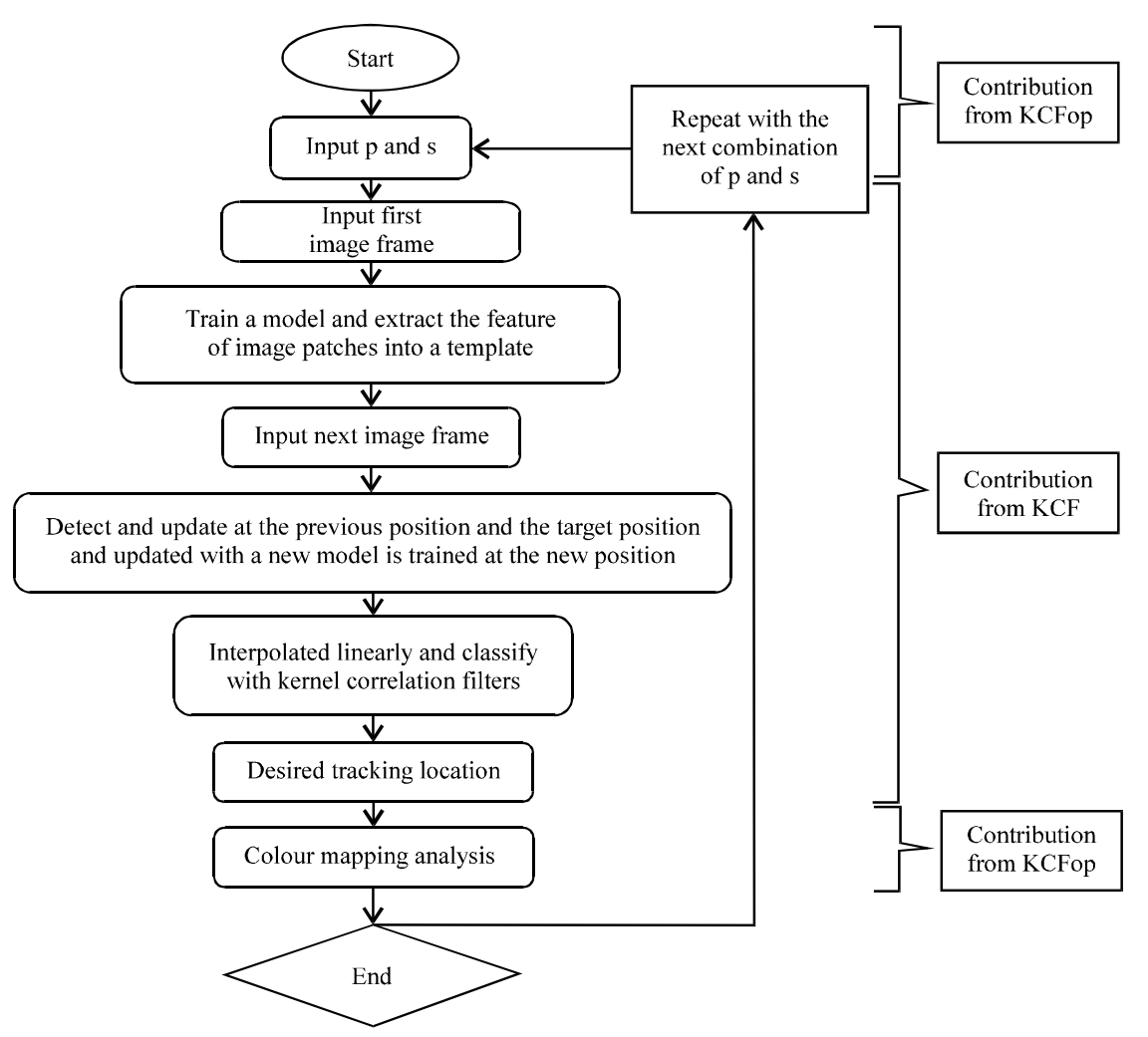

Fig. 1: The flow chart of the KCFop tracking algorithm

0.1-4.0 with increment of 0.1 while spatial bandwidth is increased from 0.01-0.4 with increment of 0.05 . The reasons of choosing 0.1 and 0.05 as the increment value for padding and spatial bandwidth, respectively are because of some limitations which are time constraint and the value of the OR and CLE are not affected even when the number of decimal is increased further for the increment value. This ensure the time to compute the tracking results to be as fast as possible.

The algorithm is run by using MATLAB 2012a with the computer's specification of Intel (R) Core (TM) i7-3520M CPU @2.90 GHz. The global best tracking results are obtained when the highest OR and the lowest CLE achieved. The analysis will be presented using colour mapping technique where the colour will provide the information each performance. In addition, the global best results are selected and compared with the others tracking methods which the OR and CLE can be obtained from the website of visual tracking benchmark under the category of TB-100 sequences.

Flow chart: The tracking pipeline is presented in this study with a flow chart shown in Fig. 1 where the main procedures conducted to perform the parameters enhancement of the visual tracking is listed out here step by step. The performance evaluator or and CLE are used to measure the tracking performance of all video sequences because OR and CLE are the most used and common performance evaluators in visual tracking and thus provide an easier way to compare with other tracking method. The techniques to calculate OR and CLE will be discussed in the following study.

Initially the padding and spatial bandwidth is set to be 1.5 and 0.1 . However, this default setting will limit the performance of the tracking. Thus, in our proposed method, padding and spatial bandwidth will be varied corresponding to frame size and the target size in the first frame for every different sequence. First, the algorithm of $\mathrm{KCF}$ is modified, so that, it can run through the different set of combinations of padding and spatial bandwidth. As the first set of combination is finished, the second combination is looped and proceeded the same as before and continue until the last combination. The tracking begins with the first sequence until the last sequences one by one.

Next, it will undergo several processes that are similar with the original $\mathrm{KCF}$ tracking algorithm where a model is trained with the image patch at the initial position of the target with the feature descriptor. Thus, a feature template is created to extract the feature of image patches. Then, 


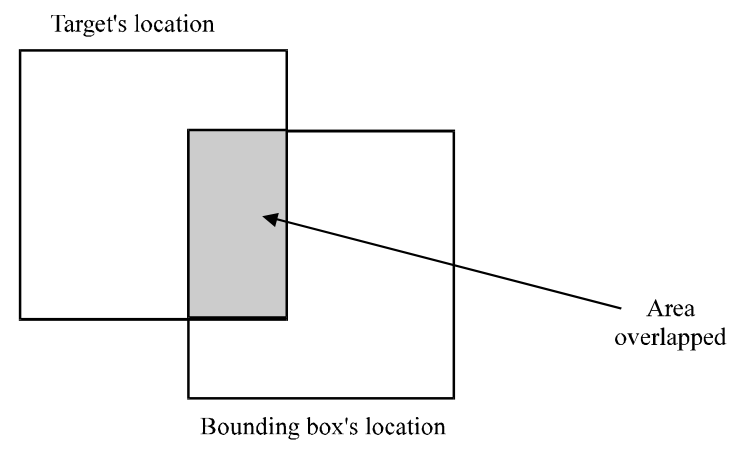

Fig. 2: Area overlapped between target's location and bounding box's location

the tracking process is started from the first frame until the last frame. The patch is detected at the previous position and the target position with maximum value of tracking performance is created and updated with a new model is trained at the new position. Based on the obtained value, it is interpolated linearly and classified with kernel correlation filters which is similar with the default algorithm by Henriques et al. (2015).

Performance evaluation: Both are qualitative evaluation and OR and CLE of our proposed method will be compared with other algorithms proposed in this visual tracking field. $\mathrm{OR}$ is defined as the percentage of the overlapping area between the region of ground truth and target as shown in Fig. 2. OR is calculated based on Eq. 1. Based on this concept or is calculated by using a MATLAB command which is "rectin" to calculate the intersecting area of these two boxes:

$$
\mathrm{AOR}=\frac{\operatorname{area}\left\{\mathrm{ROI}_{\mathrm{t}} \cap \mathrm{ROI}_{\mathrm{g}}\right\}}{\operatorname{area}\left\{\mathrm{ROI}_{\mathrm{t}} \cup \mathrm{ROI}_{\mathrm{g}}\right\}}
$$

CLE is defined as the euclidean distance between the centre location of a target size and the ground truth as shown in Fig. 3 where it is measured in pixel as shown in Eq. 2:

$$
C L E=\sqrt{\left(x_{t}-x_{g}\right)^{2}+\left(y_{t}-y_{g}\right)^{2}}
$$

This concept is basically the same with calculating the distance between 2 points using the $\mathrm{x}$ and $\mathrm{y}$ coordinate where in our case is the coordinates at the centre of the box. The higher the OR indicated that the target is tracked more accurately. OR score with more than 0.5 will only be considered as a successful tracking. While for the CLE, the lower the score it is, the better the tracking is.

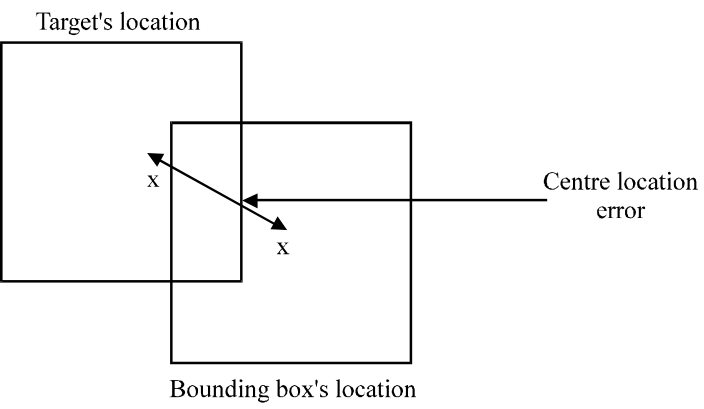

Fig. 3: CLE between target's location and bonding box's location

\section{RESULTS AND DISCUSSION}

The results obtained from the optimization and tracking performance of the proposed algorithm will be discussed in this study. A total of 86 sequences have been run through the experiment and the results of the global best performance corresponding with its own padding, $\mathrm{p}$ and spatial bandwidth, $\mathrm{s}$ are recorded. Meanwhile, the global best values of $\mathrm{p}$ and $\mathrm{s}$ obtained for each sequence are also discussed and tabulated with respect to their best tracking performance in term of $O R$ and CLE. The results of the proposed algorithm are compared with other algorithms. In this study, 15 out of the 86 sequences results are illustrated in Fig. 4.

They are Basketball, Bolt2, Boy, Car4, Coke, Crowds, David3, Deer, DragonBaby, FaceOcc1, Freemanl, Human6, Ironman, MotorRolling and Skiing in ascending order. A bounding box with red outline is plotted on the desired target location and the target will be tracked continuously with the newly updated desired tracking location until the end. Only 5 out of 12 state-of-the-art methods are depicted to be compared in these sequences where, red, green, blue, yellow, white and black represents the ground truth (desired target location), KCFop, KCF, DFT, CT and CXT, respectively.

Optimization analysis: Once, the tracking performances of one sequence is obtained, a map data study where colour scale is applied in order to determine the best tracking results. Table 2 and 3 display the colour mapping of OR and CLE, respectively for the walking sequence. In this case, there are 3 colours used as the scale in this colour mapping analysis. The best value which is corresponding to the highest $\mathrm{OR}$ and the lowest CLE will be indicated with light green while the worst value which is corresponding to the lowest $\mathrm{OR}$ and highest CLE will be indicated with dark red. The mean value between the best and the worst value will be indicated with yellow. 
(a)

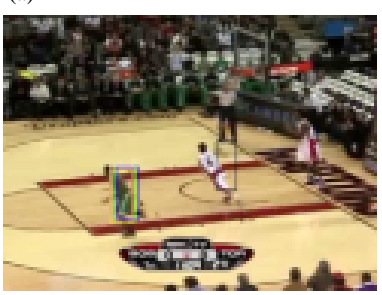

(b)

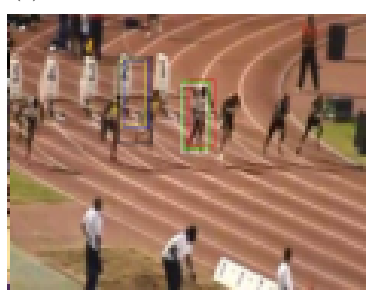

(e)
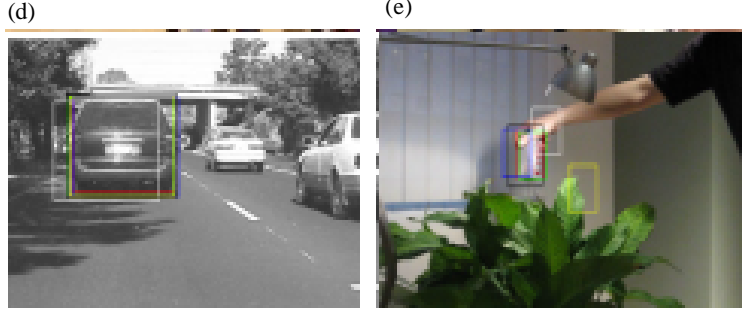

(h)
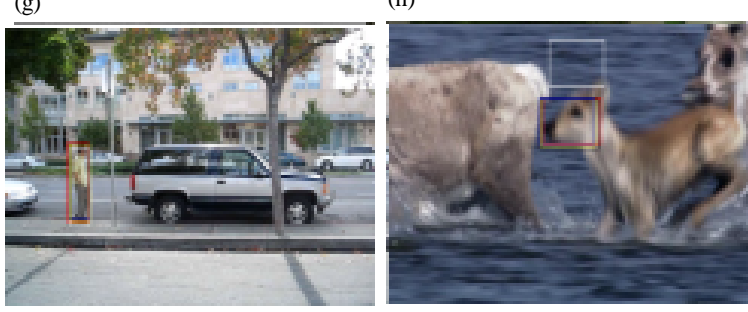

(i)
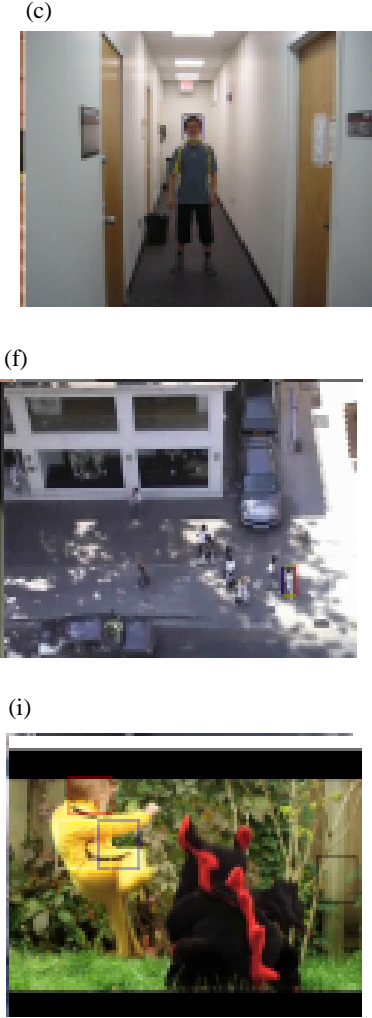

(j)

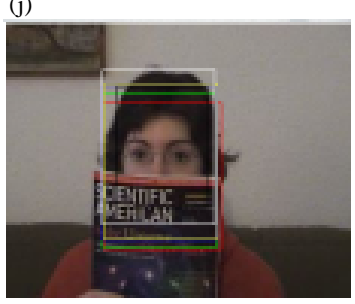

(k)
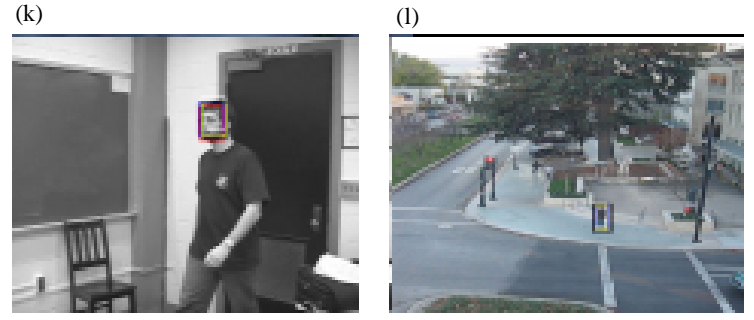

(n)

(o)
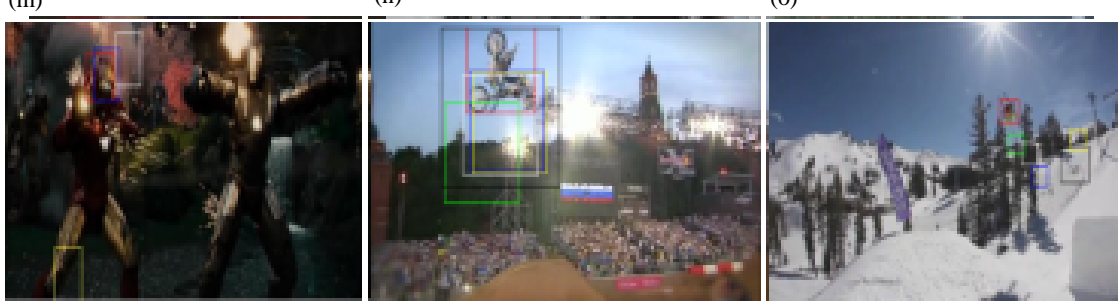

Fig. 4: a-o)Tracking performance comparisons

The density of the colour is varied through all according to the colour scale used. In walking sequence, the best tracking result of $0.486 \mathrm{OR}$ and $4.280 \mathrm{CLE}$ acquired at 1.0 padding and 0.05 spatial bandwidth. Thus, after obtaining these values of padding and spatial bandwidth, the process of analysis for the global best $O R$ and CLE with respect with its own padding and spatial bandwidth for all 86 video sequences. Once, optimization complete, the proposed algorithm, KCFop, (i.e., Kenelized Correlation Filters Optimized) results are presented in Table 4.

Tracking result: The $O R$ and CLE comparison with the $\mathrm{KCF}$ op and the other 12 tracking algorithms as tabulated 
Table 2: Color map for deciding the padding and spatial bandwith for walking sequence in term of $\mathrm{OR}$

\begin{tabular}{|c|c|c|c|c|c|c|c|c|c|c|}
\hline \multicolumn{10}{|c|}{ 84. Walking - OR } \\
\hline $\mathbf{D}$ S & 0.05 & 0.10 & 0.15 & 0.20 & 0.25 & 0.30 & 0.35 & 0.40 & 0.45 & 0.50 \\
\hline 1.0 & $\mathbf{0 . 4 8 6}$ & 0.440 & 0.250 & 0.055 & 0.017 & 0.010 & 0.010 & 0.010 & 0.010 & 0.010 \\
\hline 1.1 & 0.466 & 0.435 & 0.252 & 0.086 & 0.017 & 0.010 & 0.010 & 0.010 & 0.010 & 0.010 \\
\hline 1.2 & 0.472 & 0.457 & 0.319 & 0.101 & 0.020 & 0.012 & 0.010 & 0.010 & 0.010 & 0.010 \\
\hline 1.3 & 0.462 & 0.441 & 0.265 & 0.135 & 0.022 & 0.012 & 0.010 & 0.010 & 0.010 & 0.010 \\
\hline 1.4 & 0.475 & 0.455 & 0.327 & 0.183 & 0.048 & 0.018 & 0.011 & 0.011 & 0.011 & 0.010 \\
\hline 1.5 & 0.471 & 0.456 & 0.347 & 0.211 & 0.090 & 0.036 & 0.011 & 0.011 & 0.011 & 0.011 \\
\hline 1.6 & 0.466 & 0.445 & 0.342 & 0.220 & 0.101 & 0.037 & 0.011 & 0.011 & 0.011 & 0.011 \\
\hline 1.7 & 0.472 & 0.454 & 0.356 & 0.236 & 0.107 & 0.054 & 0.026 & 0.011 & 0.011 & 0.011 \\
\hline 1.8 & 0.459 & 0.448 & 0.350 & 0.260 & 0.105 & 0.074 & 0.027 & 0.011 & 0.011 & 0.011 \\
\hline 1.9 & 0.471 & 0.453 & 0.353 & 0.264 & 0.115 & 0.077 & 0.037 & 0.012 & 0.012 & 0.011 \\
\hline 2.0 & 0.467 & 0.456 & 0.355 & 0.288 & 0.118 & 0.086 & 0.056 & 0.011 & 0.011 & 0.011 \\
\hline
\end{tabular}

Table 3: Color map for deciding the padding and spatial bandwith for walking sequence in term of CLE

\begin{tabular}{|c|c|c|c|c|c|c|c|c|c|c|}
\hline \multicolumn{10}{|c|}{ 84. Walking - CLE } \\
\hline $\mathbf{D}$ & 0.05 & 0.10 & 0.15 & 0.20 & 0.25 & 0.30 & 0.35 & 0.40 & 0.45 & 0.50 \\
\hline 1.0 & $\mathbf{4 . 2 8}$ & 4.37 & 14.57 & 302.72 & 382.68 & 415.30 & 415.58 & 416.35 & 416.45 & 417.23 \\
\hline 1.1 & 5.17 & 4.45 & 13.96 & 219.35 & 377.06 & 415.26 & 415.26 & 415.42 & 416.12 & 416.38 \\
\hline 1.2 & 5.04 & 3.90 & 8.32 & 211.19 & 362.70 & 411.25 & 411.49 & 411.61 & 414.55 & 414.68 \\
\hline 1.3 & 5.14 & 4.34 & 13.54 & 123.77 & 362.65 & 411.25 & 415.17 & 415.14 & 415.24 & 411.71 \\
\hline 1.4 & 5.08 & 3.99 & 8.12 & 68.31 & 306.25 & 366.27 & 415.05 & 415.10 & 415.15 & 415.17 \\
\hline 1.5 & 5.12 & 3.97 & 7.25 & 40.28 & 215.55 & 310.03 & 407.21 & 414.90 & 414.17 & 414.85 \\
\hline 1.6 & 5.51 & 4.39 & 7.39 & 41.41 & 211.67 & 310.04 & 407.99 & 413.72 & 414.23 & 414.13 \\
\hline 1.7 & 5.15 & 4.09 & 6.96 & 15.65 & 207.98 & 295.81 & 327.96 & 410.06 & 412.45 & 413.03 \\
\hline 1.8 & 5.79 & 4.32 & 7.15 & 10.84 & 205.02 & 222.63 & 328.54 & 408.13 & 411.91 & 412.21 \\
\hline 1.9 & 5.16 & 4.11 & 7.04 & 10.87 & 204.76 & 219.01 & 309.96 & 403.37 & 407.82 & 409.08 \\
\hline 2.0 & 5.51 & 4.05 & 7.03 & 9.58 & 204.70 & 212.10 & 280.56 & 393.86 & 407.98 & 408.92 \\
\hline
\end{tabular}

Table 4: All 86 video sequences tracking performance of KCFop

\begin{tabular}{|c|c|c|c|c|c|c|c|c|c|c|c|}
\hline No. & Sequence & $\mathrm{p}$ & $\mathrm{s}$ & OR & CLE & No. & Sequence & $p$ & $\mathrm{~s}$ & OR & CLE \\
\hline$\overline{1}$ & Basketball & 1.8 & 0.08 & 0.761 & 6.1390 & 44 & Human4 & 1.7 & 0.07 & 0.428 & 64.9430 \\
\hline 2 & Biker & 1.5 & 0.10 & 0.243 & 77.177 & 45 & Human5 & 2.0 & 0.04 & 0.330 & 6.35900 \\
\hline 3 & Bird1 & 1.8 & 0.10 & 0.257 & 73.363 & 46 & Human6 & 1.2 & 0.05 & 0.210 & 94.6240 \\
\hline 4 & Bird2 & 0.9 & 0.05 & 0.782 & 8.2460 & 47 & Human 7 & 1.6 & 0.08 & 0.416 & 5.89700 \\
\hline 5 & BlurBody & 1.8 & 0.05 & 0.696 & 6.9420 & 48 & Human8 & 1.0 & 0.15 & 0.492 & 3.02000 \\
\hline 6 & BlurCar3 & 2.4 & 0.03 & 0.814 & 3.3340 & 49 & Humang & 1.3 & 0.10 & 0.316 & 11.4320 \\
\hline 7 & BlurFace & 1.4 & 0.04 & 0.758 & 7.2230 & 50 & Ironman & 1.5 & 0.10 & 0.145 & 194.943 \\
\hline 8 & Board & 1.2 & 0.10 & 0.715 & 14.377 & 51 & Jogging & 1.6 & 0.15 & 0.710 & 4.14200 \\
\hline 9 & Bolt & 1.4 & 0.10 & 0.682 & 6.3250 & 52 & Jump & 1.9 & 0.15 & 0.114 & 47.2740 \\
\hline 10 & Bolt2 & 2.1 & 0.25 & 0.358 & 41.030 & 53 & Jumping & 2.6 & 0.15 & 0.691 & 3.49000 \\
\hline 11 & Boy & 1.4 & 0.06 & 0.763 & 2.4440 & 54 & KiteSurf & 1.3 & 0.15 & 0.509 & 14.4270 \\
\hline 12 & Car1 & 0.9 & 0.25 & 0.121 & 39.410 & 55 & Lemming & 1.5 & 0.10 & 0.397 & 77.8680 \\
\hline 13 & Car2 & 1.4 & 0.03 & 0.685 & 3.8760 & 56 & Liquor & 1.5 & 0.10 & 0.843 & 5.26900 \\
\hline 14 & Car4 & 1.4 & 0.14 & 0.491 & 9.6850 & 57 & Man & 1.4 & 0.06 & 0.832 & 2.24300 \\
\hline 15 & CarDark & 0.4 & 0.05 & 0.777 & 2.3380 & 58 & Matrix & 1.8 & 0.25 & 0.282 & 52.2740 \\
\hline 16 & CarScale & 0.6 & 0.06 & 0.396 & 15.427 & 59 & Mhyang & 1.0 & 0.15 & 0.800 & 2.93600 \\
\hline
\end{tabular}




\begin{tabular}{|c|c|c|c|c|c|c|c|c|c|c|c|}
\hline No. & Sequence & $\mathrm{p}$ & $\mathrm{s}$ & OR & CLE & No. & Sequence & $p$ & $\mathrm{~s}$ & OR & CLE \\
\hline 17 & ClifBar & 1.8 & 0.20 & 0.456 & 10.284 & 60 & MotorRolling & 0.1 & 0.40 & 0.203 & 102.511 \\
\hline 18 & Coke & 1.7 & 0.20 & 0.703 & 9.8050 & 61 & MountainBike & 1.4 & 0.15 & 0.663 & 6.57200 \\
\hline 19 & Couple & 3.8 & 0.07 & 0.631 & 3.3860 & 62 & Panda & 1.2 & 0.07 & 0.179 & 41.0710 \\
\hline 20 & Coupon & 1.2 & 0.05 & 0.946 & 1.5320 & 63 & RedTeam & 1.2 & 0.10 & 0.478 & 3.52900 \\
\hline 21 & Crossing & 1.4 & 0.07 & 0.673 & 2.1070 & 64 & Shaking & 2.1 & 0.03 & 0.714 & 6.75700 \\
\hline 22 & Crowds & 1.5 & 0.10 & 0.794 & 3.0650 & 65 & Singer1 & 0.7 & 0.15 & 0.346 & 12.63500 \\
\hline 23 & Dancer2 & 1.6 & 0.01 & 0.742 & 5.9930 & 66 & Singer2 & 1.2 & 0.01 & 0.736 & 8.58500 \\
\hline 24 & David & 1.1 & 0.35 & 0.464 & 7.4480 & 67 & Skater & 1.2 & 0.15 & 0.572 & 10.6670 \\
\hline 25 & David2 & 1.7 & 0.11 & 0.831 & 1.9800 & 68 & Skater2 & 2.1 & 0.15 & 0.598 & 13.8200 \\
\hline 26 & David3 & 1.2 & 0.09 & 0.749 & 4.1180 & 69 & Skating1 & 2.4 & 0.15 & 0.487 & 7.44800 \\
\hline 27 & Deer & 1.3 & 0.05 & 0.660 & 8.1010 & 70 & Skating2 & 1.7 & 0.11 & 0.419 & 23.3740 \\
\hline 28 & Diving & 1.3 & 0.12 & 0.330 & 23.051 & 71 & Skiing & 1.4 & 0.30 & 0.093 & 180.515 \\
\hline 29 & Dog1 & 1.7 & 0.15 & 0.528 & 3.4340 & 72 & Soccer & 1.7 & 0.30 & 0.395 & 13.5160 \\
\hline 30 & Doll & 1.7 & 0.05 & 0.565 & 6.8320 & 73 & Subway & 1.7 & 0.15 & 0.783 & 2.46100 \\
\hline 31 & DragonBaby & 2.1 & 0.30 & 0.426 & 39.201 & 74 & Surfer & 1.8 & 0.20 & 0.439 & 3.96400 \\
\hline 32 & Dudek & 1.5 & 0.20 & 0.715 & 10.922 & 75 & Suv & 1.5 & 0.13 & 0.879 & 3.42500 \\
\hline 33 & FaceOcc1 & 2.3 & 0.03 & 0.789 & 13.406 & 76 & Sylvester & 1.8 & 0.15 & 0.623 & 12.5490 \\
\hline 34 & FaceOcc2 & 1.8 & 0.20 & 0.760 & 6.6390 & 77 & Tiger1 & 2.0 & 0.10 & 0.793 & 7.65400 \\
\hline 35 & Fish & 1.4 & 0.02 & 0.814 & 3.8670 & 78 & Tiger2 & 2.2 & 0.04 & 0.707 & 10.9740 \\
\hline 36 & FleetFace & 2.5 & 0.30 & 0.615 & 17.146 & 79 & Toy & 1.4 & 0.05 & 0.461 & 7.65300 \\
\hline 37 & Football & 1.7 & 0.40 & 0.611 & 6.6710 & 80 & Trans & 1.2 & 0.05 & 0.523 & 27.2720 \\
\hline 38 & Football1 & 2.5 & 0.10 & 0.824 & 2.1570 & 81 & Trellis & 1.9 & 0.30 & 0.595 & 7.37900 \\
\hline 39 & Freeman1 & 1.3 & 0.25 & 0.408 & 7.9080 & 82 & Twinnings & 1.4 & 0.10 & 0.590 & 4.41400 \\
\hline 40 & Freeman 3 & 1.6 & 0.10 & 0.317 & 19.254 & 83 & Vase & 1.3 & 0.09 & 0.279 & 10.5670 \\
\hline 41 & Freeman 4 & 1.6 & 0.10 & 0.391 & 4.9940 & 84 & Walking & 1.2 & 0.07 & 0.486 & 4.28000 \\
\hline 42 & Girl & 1.2 & 0.15 & 0.579 & 8.4330 & 85 & Walking2 & 0.6 & 0.15 & 0.374 & 8.52300 \\
\hline$\underline{43}$ & Gym & 1.3 & 0.10 & 0.448 & 11.844 & 86 & Woman & 1.7 & 0.10 & 0.662 & 9.66300 \\
\hline
\end{tabular}

in Table A1 and A2, respectively for all 86 video sequences. The colour of the words represents the ranking of the performance where green, blue and red colour indicate the best (highest OR), second and third place. Some of the sequences achieved significant improvement on OR and CLE but there are 6 video sequences which unable to be improved such as Biker, Crowds, Freeman3, Ironman, Lemming and Liquor.

$\mathrm{KCF}$ op has the best tracking performance in $\mathrm{OR}$ evaluation for 27 sequences, 15 sequences obtained the second highest $O R$ and 13 sequences obtained the third highest $\mathrm{OR}$. In addition, 39 sequences obtained the lowest CLE, 20 sequences obtained the second lowest CLE and 13 sequences obtained the third lowest CLE. In general, the proposed KCFop method obtained the most outstanding results in term of both average OR and CLE of all 86 video sequences which are 0.554 and 19.803 , respectively. The average OR and CLE of all 86 vedio sequences for all 13 methods are also, tabulated in Table 4 and 5 .

The improvement on average $\mathrm{OR}$ is $18.30 \%$, from 0.468 increase to 0.554 , there is significant improvement on average CLE, from 38.789 pixels reduce to 19.803 pixels which is reduced by $48.95 \%$. The result of tracking performance shows that KCFop has achieved better tracking performance followed by $\mathrm{KCF}$ and struck with both having 0.468 and then by CXT with 0.422 . KCFop has surpassed semiB the most by $125.41 \%$, followed by CT with $92.54 \%$ and lastly Frag with $67.35 \%$.
Table 5: Comparison of average OR and CLE between KCFop and the other methods

\begin{tabular}{lcclc}
\hline & OR & \multicolumn{3}{l}{ CLE } \\
Methods & Average & Different in (\%) & Average & Different in (\%) \\
\hline KCFop & 0.554 & - & 19.8030 & - \\
KCF & 0.468 & 18.30 & 38.7890 & 48.95 \\
Struck & 0.468 & 18.48 & 44.2110 & 55.21 \\
DFT & 0.343 & 61.45 & 78.6000 & 74.81 \\
CSK & 0.399 & 38.70 & 91.5060 & 78.36 \\
ML & 0.350 & 58.51 & 65.2640 & 69.66 \\
Frag & 0.331 & 67.35 & 78.8090 & 74.87 \\
CT & 0.288 & 92.54 & 77.4310 & 74.42 \\
OAB & 0.357 & 55.16 & 71.9730 & 72.49 \\
LOT & 0.335 & 65.20 & 63.3740 & 68.75 \\
CXT & 0.422 & 31.26 & 66.8110 & 70.36 \\
semiB & 0.246 & 125.41 & 175.495 & 88.72 \\
VTD & 0.387 & 43.20 & 53.6630 & 63.10 \\
\hline
\end{tabular}

KCFop also, acquired the lowest average CLE among the other methods, followed by KCF with 38.789 pixels and later by struck with 44.211 pixels. Furthermore, based on the CLE percentage difference, KCFop has also, outperformed semiB by $88.72 \%$, followed by CSK with $78.36 \%$ and lastly Frag with $74.87 \%$. Thus, KCFop had achieved a better improved and able to obtain a promising tracking performance in our proposed method (Appendex)

\section{CONCLUSION}

The proposed method, KCFop has promising tracking performance among the 12 tracking methods as compared in here. There are still having many challenging issues 
due to the destabilizing attributes that lead to the performance drift. Overall, the proposed concept of simple optimization that is implemented into the tracking algorithm by enhancing the global best padding and spatial bandwidth obtained. Through this parameters optimization, the average OR of the proposed method is increased to 0.554 with $18.30 \%$ of improvement and the average CLE of the proposed method is decreased to 19.803 pixels with $48.95 \%$ of improvement as compared to the $\mathrm{KCF}$ when tested on 86 video sequences. Thus, KCFop is commended to enhance visual tracking.

\section{ACKNOWLEDGEMENT}

This research and publication were supported by Universiti Sains Malaysia Grant No. 1001/PAERO/ 814276.

\section{NOMENCLATURE}

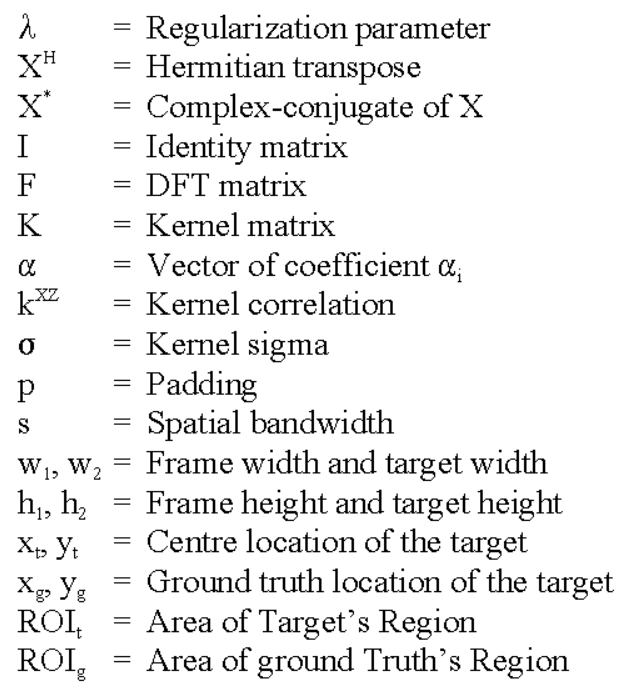

\section{Appendix:}

Table Ala: Comparison of OR between KCFop with other algorithms

\begin{tabular}{|c|c|c|c|c|c|c|c|c|c|c|c|c|c|c|}
\hline & & Fop & $\mathrm{CF}$ & Tuc & DFT & $\operatorname{CSK}$ & MIL & Frag & CT & OAB & \begin{tabular}{|l|} 
LOT \\
\end{tabular} & $\mathrm{CXT}$ & $\mathrm{min}$ & $\mathrm{TD}$ \\
\hline 1 & Basketball & 761 & 676 & 2003 & 607 & $|0.706|$ & 220 & 0619 & 0256 & 0020 & 0681 & 0023 & 022 & 727 \\
\hline 2 & ker & 243 & 243 & 266 & 251 & 257 & 029 & 0225 & 013 & 0260 & 285 & & & \\
\hline & & & 054 & 2 & 186 & 018 & & 0.042 & & 0055 & 035 & & & $m$ \\
\hline 4 & & & 15 & 567 & 18 & 578 & & 435 & 096 & 0.648 & 0084 & 230 & 363 & \\
\hline 5 & $g d y$ & 696 & 435 & .724 & 096 & 0.443 & 0030 & 0543 & 0,030 & 0.713 & 0263 & 0.729 & 01 & 0236 \\
\hline 6 & burCar3 & 814 & .788 & 773 & 121 & $0.4 S 2$ & 0273 & 0.458 & 0219 & 0.730 & 0240 & 0601 & 0.725 & 0.187 \\
\hline 7 & BhuFace & 758 & 0.737 & 0.469 & 0328 & 0.778 & 0275 & 0642 & 0.151 & 0.481 & 0.198 & 0.833 & 0.469 & 0.416 \\
\hline 8 & & & 639 & & 325 & 0.444 & 0.407 & 0512 & \begin{tabular}{|l|l|}
0.447 \\
\end{tabular} & 0265 & 0200 & 0290 & 200 & 0266 \\
\hline 9 & & & 679 & 14 & 0034 & 0019 & 0011 & 113 & 0000 & 0042 & 21 & 0016 & 159 & 0366 \\
\hline & 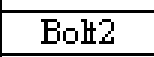 & & 1 & 222 & 10 & 0.481 & 683 & 0325 & 0367 & 11 & 514 & 0012 & 0,031 & 0502 \\
\hline 11 & & 63 & 0.750 & 760 & 0.401 & 0657 & 0.491 & 0389 & 0590 & 0.791 & 0533 & 0542 & 0323 & 0625 \\
\hline 12 & arl & 21 & 0.100 & .111 & 0096 & 0098 & 0093 & 0082 & 0066 & 0083 & .141 & \begin{tabular}{|l|}
0.397 \\
\end{tabular} & 0056 & \begin{tabular}{|l|}
0.118 \\
\end{tabular} \\
\hline 13 & $\overline{a r 2}$ & 685 & 0658 & 0688 & 0.157 & 0,690 & 0.166 & 259 & 0234 & 0569 & 0.104 & 0.875 & 0.493 & 801 \\
\hline 14 & $\operatorname{ar4}$ & 91 & 480 & 489 & 237 & 0.466 & 0258 & 188 & 0213 & 0213 & 0042 & 0307 & 0225 & 0360 \\
\hline 15 & apak & 777 & 9 & 892 & 380 & 0.755 & 0.195 & 301 & 0,003 & 0.785 & 0.421 & 0566 & 1.832 & 05 \\
\hline 16 & $\mathrm{Cars}$ sale & 196 & 0380 & 1.412 & 14 & 0.415 & 0.410 & 0.425 & 0,431 & 0395 & 0346 & 0.684 & 0323 & 0.430 \\
\hline 17 & $\mathrm{fBar}$ & 156 & 0244 & 0.478 & 199 & 0,495 & 0.489 & 0.174 & \begin{tabular}{|l|l|}
0.447 \\
\end{tabular} & 0548 & 0247 & 0520 & 0.163 & 0.435 \\
\hline 18 & ke & 03 & 0549 & 673 & 0.100 & 0570 & 0200 & 0039 & 0225 & 0330 & 0.122 & 0.423 & 0046 & 0.142 \\
\hline 19 & suple & 31 & 0.190 & 536 & 0077 & 0075 & 0.498 & 569 & 0.46 & 0358 & 0.448 & 0.483 & 0342 & 0,064 \\
\hline 20 & up & 46 & 0. & 0876 & $b$ & 0898 & 0623 & 0251 & 0574 & 0505 & 0218 & 0833 & 0.148 & 0645 \\
\hline 21 & ossing & 0.673 & 0.664 & 0677 & \begin{tabular}{|l}
0.496 \\
\end{tabular} & 0.479 & 0.727 & 0311 & \begin{tabular}{|l|}
0.683 \\
\end{tabular} & 0664 & 0303 & 0361 & 0.684 & 031 \\
\hline 22 & owds & & 794 & 613 & 793 & 0.761 & 0055 & 0,023 & 0004 & 0074 & 001 & 0093 & 0071 & 002 \\
\hline- & Danoen2 & & 0.716 & .762 & 792 & 0.788 & 0.734 & 0.741 & 0.736 & 0.752 & 0691 & 0.724 & 0540 & 0.72 \\
\hline 24 & avid & 64 & \begin{tabular}{|l}
0.431 \\
\end{tabular} & 239 & 0297 & 0.402 & 0.428 & 0.169 & \begin{tabular}{|l|}
0.496 \\
\end{tabular} & 0387 & 0264 & $0.649^{9}$ & 0243 & 558 \\
\hline 25 & David2 & 31 & 0827 & 871 & 0541 & 0820 & 0.451 & 0240 & 0000 & 0318 & 0602 & 0.883 & 0536 & 0686 \\
\hline 26 & vid3 & & \begin{tabular}{|l|}
0.740 \\
\end{tabular} & 0292 & 0559 & 0.498 & 0537 & 70 & 0306 & 0326 & 0.665 & 0.120 & 0.149 & 0.403 \\
\hline 27 & & 0660 & 0595 & 0.740 & 0256 & 0.745 & 0.124 & 0.173 & 0040 & 0.721 & 0.198 & 0697 & 0639 & 058 \\
\hline & & & & & & & & & & & & & . & \\
\hline
\end{tabular}


Table Alb: Comparison of OR between KCFop with other algorithms

\begin{tabular}{|c|c|c|c|c|c|c|c|c|c|c|c|c|c|c|}
\hline 4 & $\operatorname{og} l$ & 5 & 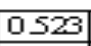 & & & & & & $-\infty$ & 544 & & & & \\
\hline 30 & 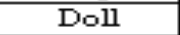 & 5 & 50 & 45 & 75 & 16 & 166 & 34 & & $3-6$ & & & 22 & \\
\hline 31 & rag & 26 & 315 & 201 & 141 & 203 & 254 & 8 & $\infty$ & 190 & & & & \\
\hline 32 & & 15 & 698 & 7300 & 690 & 716 & & 535 & 77 & 657 & & & & \\
\hline 3 & & & 72 & 20 & 87 & 5 & & & & & & & & \\
\hline & & & & & & & & & & & & & & \\
\hline 35 & & & & & 57 & & & & & 37 & & & & \\
\hline 36 & Pao & & 546 & & 482 & 587 & & 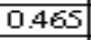 & 321 & & & & & \\
\hline 37 & & & 502 & 534 & 658 & 553 & 580 & 701 & 10 & 1335 & & & 153 & \\
\hline & & & 576 & 668 & & 0.465 & 660 & 364 & 227 & & & & 125 & \\
\hline 可 & & & 191 & 338 & 386 & 5 & 341 & 374 & 144 & 03 & & & & \\
\hline 0 & & & 317 & & & & & & & & & & & \\
\hline 41 & $\tan$ & & & 미 & 8 & & 54 & 41 & 105 & $\theta$ & & & 83 & \\
\hline-1 & & & & 45 & & & 8 & 4 & & & & & & \\
\hline 43 & & & 153 & +281 & 111 & & & & & & & & & \\
\hline 44 & & 288 & 54 & 142 & 135 & 0.138 & 122 & 0.050 & 0.128 & 1.135 & 143 & & 078 & \\
\hline 45 & & & & & & & 213 & 030 & & & & & & \\
\hline 5 & & & 3 & 214 & 7 & & 2 & & & 0204 & & & & \\
\hline & & & 56 & & & & & & & & & & & \\
\hline 18 & & & 57 & 2 & 119 & 184 & 23 & 97 & 35 & 97 & & & 21 & \\
\hline 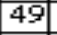 & & & $\mathrm{II}$ & & & 248 & & & & 11 & & & 4 & \\
\hline 50 & & & & & & & & & & 8 & & & 37 & \\
\hline 51 & Jogging & & 181 & 171 & 87 & 0. & 181 & 0518 & 8 & 0 & 92 & & 530 & 54 \\
\hline-1 & & & 064 & 10 & & & 54 & 75 & & & & & 40 & 60 \\
\hline & & & 279 & 517 & & & 21 & & & & & & 88 & \\
\hline & & & 44 & & & & 312 & 35 & 37 & 14 & & & & \\
\hline & & & 97 & 8 & & & & & & & & & 15 & \\
\hline & & & & 12 & & & & & & & & & 76 & \\
\hline 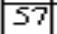 & & & & & 202 & & 194 & 0201 & 0227 & 0859 & & & & \\
\hline 01 & & & 119 & 997 & 58 & 31 & 22 & 57 & & & & & & 21 \\
\hline & & & & & & 794 & & 651 & & & & & 64 & 0.731 \\
\hline
\end{tabular}

Table Alc: Comparison of OR between KCFop with other algorithms

\begin{tabular}{|c|c|c|c|c|c|c|c|c|c|c|c|c|c|c|}
\hline 00 & $10 \mathrm{NT}$ & & $0 \mathrm{~S}^{-1}$ & & 084 & 090 & 122 & 118 & & 0.110 & 0.121 & .129 & 003 & \\
\hline 61 & $6 y$ & & 627 & & 298 & & 52 & 28 & & 31 & 0578 & & 02 & \\
\hline 52 & & & 150 & & 279 & & & & & & 041 & & & \\
\hline & & & 467 & & 272 & & & 138 & & 12 & $\overline{10}$ & & $=$ & 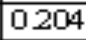 \\
\hline 7 & & & 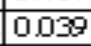 & & 637 & & 427 & 082 & & & .125 & & 007 & .71 \\
\hline 65 & & & 331 & 58 & 355 & & 0356 & 205 & 0348 & 336 & 0.194 & 92 & 0.117 & .488 \\
\hline 66 & & & & & & & & & & & 256 & & & 417 \\
\hline 67 & & & & & & & 13 & & 80 & 532 & 445 & & & - \\
\hline & & & Q & & 168 & & 80 & 45 & 45 & 32 & 54 & & 20 & גas \\
\hline 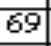 & & & 6 & & 135 & & 27 & & & 394 & 0258 & & 084 & 52 \\
\hline 70 & & & 341 & & 149 & & 0.175 & 205 & 214 & 0240 & .414 & & 0.055 & .440 \\
\hline 71 & & & 47 & & 51 & & 55 & 33 & & & 21 & & 43 & $=8$ \\
\hline 72 & & & 362 & & 16 & & .171 & 74 & 39 & 86 & 03 & & 0006 & $\Rightarrow$ \\
\hline 73 & & & $\pi$ & & 25 & & & & & 64 & 0562 & & 0286 & 57 \\
\hline 74 & & & 3800 & & 042 & & 0250 & 0221 & 0088 & 1039 & 0322 & & 0.103 & 318 \\
\hline 75 & & & 878 & & 072 & & 0.194 & 0.628 & 0235 & 1628 & 0.654 & & 0378 & 1.453 \\
\hline 76 & & & 607 & & 377 & & 0 & 24 & & 60 & 55 & & 41 & 19 \\
\hline \begin{tabular}{|l|}
77 \\
\end{tabular} & & & 782 & & 532 & & 0.120 & 0265 & .410 & 100 & 0.130 & 22 & 0.400 & 1.118 \\
\hline 78 & & & 51 & & $\pi$ & & 55 & 18 & 42 & 51 & 35 & & 53 & 303 \\
\hline 79 & & & 425 & & 431 & & 0230 & 80 & 0362 & 0297 & 0299 & & 0.143 & 0527 \\
\hline 80 & & & 389 & & 0.488 & & 034 & 0 & 11 & 1503 & 0.461 & & 0.137 & 529 \\
\hline 81 & & & 522 & & 0361 & & 0248 & 85 & 36 & 40 & 06 & & 0.198 & 1.4 \\
\hline 82 & & & 0550 & & \begin{tabular}{|l|l|}
0.485 \\
\end{tabular} & 0522 & 0512 & \begin{tabular}{|l}
0.471 \\
\end{tabular} & 0.444 & 0.420 & 0504 & 36 & \begin{tabular}{|l|l|}
0272 \\
\end{tabular} & 6 \\
\hline 83 & & & 0277 & & & & & & & 1262 & & & .117 & 0282 \\
\hline 84 & & & 0.456 & & 0562 & & 0545 & 0537 & 0520 & 0543 & 04 & 71 & 0.194 & 0.607 \\
\hline 85 & & & 279 & & 405 & & 0288 & 0274 & 0266 & 0363 & 0335 & 69 & 63 & 0325 \\
\hline \multirow[t]{2}{*}{86} & & & 0.658 & & 756 & & 57 & 47 & 31 & 0.479 & 0089 & & 100 & 44 \\
\hline & F & & & & & & & & & & & re & 0.240 & \\
\hline
\end{tabular}


Table A2a: Comparison of CLE between KCFop with other algorithms

\begin{tabular}{|c|c|c|c|c|c|c|c|c|c|c|c|c|c|c|}
\hline & & CFQD & $\mathrm{KCF}$ & & 2.1 & $\operatorname{csK}$ & MIL & Frag & $\mathrm{CT}$ & OAB & LOT & CXT & $28 x$ & VTD \\
\hline & . & 39 & 889 & 1825 & .028 & 27 & .918 & & 106 & 5.4 & 678 & & 98 & -8 \\
\hline 2 & & 177 & 1.177 & & & & & & & & & & & \\
\hline 3 & & 363 & 52340 & & & 730 & & & & & & & & \\
\hline 4 & & & 21.370 & 747 & 7779 & 297 & & 8.100 & & & & 42.898 & & 46 \\
\hline 5 & BhorF & 48 & 4.115 & & & 0.666 & 65.746 & 37.794 & 76 & 54 & $\mathrm{jl}$. & 99590 & 14 & 69 \\
\hline 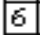 & harCar3 & 34 & 138 & & 4 & 66813 & $8.15 \mathrm{~s}$ & 58.218 & 68.566 & 5.110 & 00645 & 5.154 & 44.29 & 97. \\
\hline 7 & Bhar] & 223 & 364 & 2.353 & 5.636 & 9681 & 1.976 & 3.618 & 195 & 54.388 & 66877 & 6.44 & & 0.669 \\
\hline 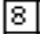 & & 3 & 137 & 02 & 8.862 & 5.934 & 1.819 & 91.842 & 3.051 & 113606 & 82389 & 125.42 & 350.4 & $25 x$ \\
\hline 2 & & 325 & 365 & 803 & 36727 & 20395 & 393543 & 18338 & 363.79 & 256281 & 12512 & 385 & s & 58 \\
\hline 10 & $\overline{\mathrm{B}}$ & 1.030 & 329.808 & 86.408 & 76.700 & 1.634 & 7.392 & 3.208 & $15.19 \mathrm{G}$ & 136575 & 399 & 266.15 & 27708 & .115 \\
\hline 11 & & 444 & 867 & & & & 12.835 & & 9031 & 2.917 & 66.002 & 7392 & 211.79 & 74 \\
\hline 12 & & 410 & 42435 & 51.720 & 55 & 70.435 & 112.169 & 115.71 & 741 & 5.249 & 114.463 & 20.748 & 13 & 110068 \\
\hline 13 & & & & 2.418 & & & & 99 & & & & & 44.925 & \\
\hline 14 & & 685 & 9 & 8 & & 34 & 77 & 131549 & 27 & 325 & 85 & 118 & 15225 & 95 \\
\hline 13 & $=3$ & 3388 & 6 & 0.954 & 16 & 233 & 43.478 & 66 & 19 & 2838 & 353 & 90 & 84 & \\
\hline 10 & $\mathrm{C}$ & 15.427 & 6.142 & 36.431 & 52 & 115 & 33.471 & 13 & 953 & 739 & 10122 & 19 & 13204 & 38.461 \\
\hline 17 & C & 10.284 & 721 & 23.507 & 3.984 & 1877 & 1.367 & 3 & 80 & 7.820 & 25 & 舟 & 1087 & 24.66 \\
\hline 18 & ke & 5 & 653 & 85 & 98 & 14 & 24 & $4 x$ & 491 & 856 & 20 & 25.740 & 365276 & 68.653 \\
\hline 19 & Couple & 3886 & .556 & 3 & 859 & - & 4.526 & 8.801 & 35 & 21 & 902 & 41.758 & 38 & 042 \\
\hline & $m$ & 37 & 1568 & 13 & 518 & 40 & 666 & 9 & 18.98 & 15 & 84 & 93 & 11881 & 57 \\
\hline t & $\mathrm{me}$ & 107 & 2.250 & 08 & 200 & 8959 & 177 & 38.589 & 3560 & 40 & 36.713 & 23.414 & 645 & 66.125 \\
\hline 2 & $\mathrm{C}$ & 68 & 3.065 & 328 & 331 & 691 & 428345 & 7 & 379 & 2 & 94 & 27754 & 499 & 47 \\
\hline 5. & Danoeñ & 993 & 12 & 911 & 1 & 857 & 9.732 & 11.235 & 8597 & 10.204 & 15.643 & 7.442 & 74.124 & 7870 \\
\hline 24 & id & 148 & 162 & .801 & 876 & 80 & .860 & 82.071 & 0.494 & 21.733 & 23.854 & 6.048 & 47.002 & 11.586 \\
\hline 25 & David2 & 1.980 & 2083 & 199 & 7.288 & 2320 & 0.927 & 56.868 & 76.704 & 33.007 & 4092 & 1.321 & 11.849 & 2856 \\
\hline 26 & David3 & 18 & 302 & 650 & 0.931 & 56.102 & 9.681 & 13.552 & 88.664 & 83.435 & 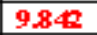 & 222212 & 236.46 & 66.722 \\
\hline 27 & eer & 101 & 1.165 & 270 & 8.749 & 973 & 100.729 & 10508 & 246.42 & 6980 & 65.223 & .749 & 61.531 & 134849 \\
\hline 28 & Diving & 3.051 & 42.571 & 19.227 & 0.572 & 81.773 & 62.677 & 84.745 & 98.051 & 55.586 & 37.272 & 64.275 & 17757 & 73.819 \\
\hline 29 & Dogl & 134 & & 5663 & 41.237 & & 7832 & 11.921 & 6986 & 5897 & 4635 & 4886 & 75.419 & 11.038 \\
\hline 30 & Ill & & 5 & 19 & 15 & 721 & 77 & 38 & 24 & 2.300 & 22 & 1.654 & 113 & 294 \\
\hline
\end{tabular}

Table A2b: Comparison of CLE between KCFop with other algorithms

\begin{tabular}{|c|c|c|c|c|c|c|c|c|c|c|c|c|c|c|}
\hline & DragonBaby & 201 & 0.399 & 255 & 5.583 & 87.900 & 3.611 & 46.387 & 9.040 & 80.062 & 6.821 & 40.737 & 5.482 & 41.425 \\
\hline & Dudek & 0292 & 1.382 & 1.449 & 18.719 & 13.394 & 7.697 & 82.603 & & & 5.125 & 12.818 & & 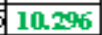 \\
\hline & & & & & & & & & & & & & & \\
\hline & Fac & & 66 & 5 & 75 & & & & & & & 29 & & \\
\hline & Fish & & $\pi$ & 401 & 8838 & & & & & & & & & \\
\hline & Fles: & & 370 & & .016 & & 19 & 7.650 & & 97 & & & & 16.134 \\
\hline & Footl & & 14.602 & 17.312 & 9293 & $\mid 16.187$ & 2.087 & 7 & & 72.371 & 53 & 12.863 & 361 & 13.640 \\
\hline & Footh & & 5.474 & 5.465 & 1.975 & 16.51 & 5618 & 15.607 & 0.712 & 85.677 & 6850 & 11 & 2035 & 7.437 \\
\hline & Freen & & 94.883 & 14.275 & 10.432 & 125.464 & 11.216 & 10.097 & 18.716 & 35.722 & 86.911 & 26.847 & 174354 & 10.317 \\
\hline & Free & .254 & 2.254 & 834 & 2.602 & 53.900 & 87.565 & 40.467 & 65.319 & 40.661 & 40.527 & 3593 & 52661 & 23.957 \\
\hline & Fre & & 7.116 & 606 & 57.478 & 0 & 9 & 88 & 86 & 13593 & & & 83 & 1.68 \\
\hline & $\mathrm{G}$ & & 16 & 73 & 23.981 & 12 & 66 & 20 & 52 & 96 & 83 & 55 & 52 & 98 \\
\hline & Gym & & 5.258 & 3.463 & 104.4 & 27 & 11.798 & 39 & 43 & 164 & 9.171 & 33 & 30366 & 10.84 \\
\hline & $\mathrm{Hu}$ & 943 & $\pi$ & 253.18 & 2459 & 21820 & 24.1 & $0 \Delta$ & $906 \mathrm{~s}$ & 174.13 & $2 \theta$ & 2737 & $100>2$ & 162.14 \\
\hline & $\mathrm{H}$ & & 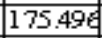 & 6.873 & (2) & 200. & 21. & 2862 & 40.82 & 89.13 & 02 & 00861 & 02.70 & 1.249 \\
\hline rat & Humaró & .024 & 107652 & 87.038 & We & 19004 & 35.374 & 84.193 & 60.175 & $x+\infty$, & 35.866 & 96.366 & (41) & 120,00 \\
\hline & $\mathrm{Hum}$ & & 48.198 & 4557 & 021 & 87 & 4.047 & 44.123 & 71 & 14.365 & 29 & 9 & 132994 & Qu \\
\hline & & & 843 & 3.786 & & & 74.947 & 74.834 & 92.137 & 74.153 & & 24 & & 99 \\
\hline & $\theta$ & & 1769 & 42.454 & 56.440 & 44.698 & 23.307 & 37 & 29.670 & 24.179 & 73 & 48.394 & 631 & 33.4 \\
\hline & $I_{x \rightarrow}$ & & 9494 & 127.670 & 2396 & 18 & 193374 & 270874 & $E$ & 21380 & & 162672 & - & 63.248 \\
\hline & Jog & & 88.257 & .055 & 31.443 & 134984 & 96.345 & 21.457 & 92.494 & 164 & 90.876 & 5590 & 51.985 & 83.290 \\
\hline & p & 274 & 84.114 & 84.828 & 92.602 & 790 & 135.72 & 78.120 & 35 & 11060 & 41.289 & 132.196 & 227.149 & 14693 \\
\hline 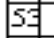 & humping & & 26.117 & 6547 & 67.062 & 85.972 & 9992 & 5.602 & 47.731 & 46.345 & 5575 & 9991 & 94.258 & 41.387 \\
\hline & KiteSurf & & 17.269 & 6.135 & 32.397 & 36.474 & 22.601 & 141.139 & 90.063 & 64.598 & 84.386 & 36.773 & 32.856 & 76.258 \\
\hline & Lerrming & 77.868 & 77.868 & 37.753 & 77.754 & 114237 & 12065 & 12687 & 32.252 & 18.051 & 19.116 & 61.303 & 286245 & 79.224 \\
\hline & Liquor & & 5. & 90.995 & 221.128 & 160 & 14188 & 99.647 & $185 \&$ & 71.074 & 30 & 131805 & 231344 & 60.172 \\
\hline & & & 2259 & 1.369 & 39.939 & & 36.704 & 57.023 & 35.067 & 2319 & 33 & 2235 & 47 & \\
\hline & & & 76.423 & 194545 & 105.784 & 13692 & & & 129.108 & 10885 & & 51587 & 85.492 & 76.735 \\
\hline & Ml & & 3922 & & 063 & & & & & & & & 4.272 & 43 \\
\hline & & & & & & & & & & & & & & \\
\hline
\end{tabular}


Table A2c: Comparison of CLE between KCFop with other algorithms

\begin{tabular}{|c|c|c|c|c|c|c|c|c|c|c|c|c|c|c|}
\hline \multicolumn{2}{|c|}{ 61 MountainBik } & & 661 & 625 & 15016 & & & -1 & & .006 & & & & \\
\hline 62 & Panda & & 2055 & .181 & 48.457 & & 5948 & 655 & 9.000 & 3362 & 297 & 4.022 & 602 & 4.600 \\
\hline 票 & $\mathrm{R}$ & 529 & 81 & 275 & 0.251 & 3.033 & 6651 & 0 & & 803 & 662 & 806 & 192 & 2.9 \\
\hline 6 & & & & 8 & .292 & & 24.027 & 2.146 & 017 & & .595 & $\overline{20}$ & 43 & 9.046 \\
\hline 6. & & & 5 & 1.514 & 8.784 & & 16.365 & 8.869 & 834 & 2.928 & 389 & 11.361 & & 7 \\
\hline & & & & 33 & 48 & & 22.530 & 34 & & 87 & 76.837 & 30 & 74 & 43.690 \\
\hline 6 & & 667 & 100 & \begin{tabular}{|l|l|}
8.204 \\
\end{tabular} & 6.034 & 1 & 10.202 & 4.253 & 06 & 20.609 & 9.499 & 7.622 & 168 & 9.737 \\
\hline & & & 17.901 & & 125 & & 35.372 & 0.016 & 56 & 4.419 & & 26.465 & 74 & 22.351 \\
\hline & & & 6 & 63 & & & 130 & 49 & & & & & & 347 \\
\hline 70 & $S$ & & 30.762 & 7.883 & 139 & 22 & 108 & 125 & 73.728 & 4.814 & 54 & 1706 & 336. & \\
\hline 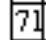 & & & & & 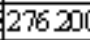 & & & & & & & & & 632 \\
\hline 72 & & 516 & 15.375 & 360 & 13953 & 70 & 77.853 & 55 & 97 & 12753 & 37 & 9.216 & $2 \Delta \triangle D$ & 23.557 \\
\hline 7 & & & & 469 & & & 7595 & 92 & & & 13 & 308 & II) & 3. \\
\hline 74 & & & 8.737 & 9043 & 1 & & 16.960 & 51.593 & 35.104 & 72.064 & 26.000 & 3.072 & & .272 \\
\hline 然 & & & 3.4 & 0.622 & 112 & 57 & 65 & 01 & 669 & & 78 & 9.891 & $1 \Delta J$. & 247 \\
\hline 76 & lv & 2.549 & 12.918 & 6.299 & 44.883 & 16 & 15.204 & 14.996 & 8556 & 14.807 & 11.350 & 14.782 & R日 & 19.601 \\
\hline 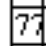 & & & & 8697 & 41.298 & 3 & 10892 & 45 & 29 & 81 & 111375 & 61 & 10525 & 10727 \\
\hline 78 & & 4 & 4 & 78 & 28 & & 27 & 42 & 28.374 & 663 & 15 & 41.437 & tor & 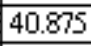 \\
\hline 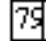 & & & + & 371 & 31.882 & 3.005 & 58.059 & 27.428 & 22.431 & 3.301 & 53.031 & 120 & 15322 & 10.628 \\
\hline$=$ & & & 54.675 & 3.186 & 56.155 & & 89.033 & 62 & & 5.200 & 59.961 & 67.228 & 400 & 45 \\
\hline 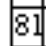 & & & 7.764 & 6917 & 44.872 & 8.824 & 71.469 & 59.508 & 41.695 & 98.330 & 47.738 & 7.006 & 69.042 & 32.250 \\
\hline 82 & $2 x$ & & 6.767 & 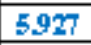 & 12.777 & 9973 & 9853 & 61 & 88 & 17.765 & 20.959 & 21.861 & 11125 & 6.253 \\
\hline 83 & & & 12488 & 24.276 & 13.250 & 12879 & 18.953 & 18.168 & 15.827 & 34.694 & 17.500 & 14.118 & 17228 & 19.635 \\
\hline 84 & & 280 & 971 & 4632 & 5870 & 7.169 & 3.397 & 9306 & 6950 & 5330 & 2 & 20567 & 32700 & 5.783 \\
\hline 83 & Wall & & 28.983 & 163 & 29.006 & 17.931 & 60.647 & 57.525 & 58.526 & 29.108 & 64.866 & 34.689 & 12288 & 46.245 \\
\hline 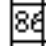 & - & & 10.063 & 68 & 98 & 207336 & 12529 & 11924 & 114.482 & 32.277 & 130903 & 72.492 & 1154 & 11886 \\
\hline & & & & & & & & & & & & & & \\
\hline
\end{tabular}

\section{REFERENCES}

Adam, A., E. Rivlin and I. Shimshoni, 2006. Robust fragments-based tracking using the integral histogram. Proceedings of the 2006 IEEE Computer Society Conference on Computer Vision and Pattern Recognition (CVPR'06), June 17-22, 2006, IEEE, New York, USA., ISBN:0-7695-2597-0, pp: 798805.

Avidan, S., 2004. Support vector tracking. IEEE Trans. Patt. Anal. Mach. Intel., 26: 1064-1072.

Babenko, B., M.H. Yang and S. Belongie, 2009. Visual tracking with online multiple instance learning. Proceedings of the International Conference on Computer Vision and Pattern Recognition, June 20-25, 2009, Miami, FL., pp: 983-990.

Bao, C., Y. Wu, H. Ling and H. Ji, 2012. Real time robust L1 tracker using accelerated proximal gradient approach. Proceedings of the 2012 IEEE Conference on Computer Vision and Pattern Recognition (CVPR), June 16-21, 2012, IEEE, Providence, Rhode Island, ISBN:978-1-4673-1226-4, pp: 1830-1837.
Bolme, D.S., J.R. Beveridge, B.A. Draper and Y.M. Lui, 2010. Visual object tracking using adaptive correlation filters. Proceedings of the 2010 IEEE Computer Society Conference on Computer Vision and Pattern RecognitionIn (CVPR), June 13-18, 2010, IEEE, San Francisco, California, ISBN:978-1-4244-6984-0, pp: 2544-2550.

Chen, S., S. Li, R. Ji, Y. Yan and S. Zhu, 2016a. Discriminative local collaborative representation for online object tracking. Knowl. Based Syst., 100: 13-24.

Chen, W., K. Zhang and Q. Liu, 2016b. Robust visual tracking via patch based kernel correlation filters with adaptive multiple feature ensemble. Neurocomput., 214: 607-617.

Cheng, X., N. Li, T. Zhou, L. Zhou and Z. Wu, 2015. Object tracking via collaborative multi-task learning and appearance model updating. Appl. Soft Comput., 31: $81-90$.

Dalal, N. and B. Triggs, 2005. Histograms of oriented gradients for human detection. Proceedings of the 2005 IEEE Computer Society Conference on Computer Vision and Pattern Recognition (CVPR'05), June 20-25, 2005, EEEE, SanDiego, California, ISBN:0-7695-2372-2, pp: 886-893. 
Dinh, T.B., N. Vo and G. Medioni, 2011. Context tracker: Exploring supporters and distracters in unconstrained environments. Proceedings of the 2011 IEEE Conference on Computer Vision and Pattern Recognition (CVPR), June 20-25, 2011, IEEE, Colorado Springs, Colorado, ISBN:978-1 -4577-0394-2, pp: $1177-$ 1184.

Fan, B., Y. Du and Y. Cong, 2014. Online learning discriminative dictionary with label information for robust object tracking. Abstract Appl. Anal., 2014: 112.

Fergus, R., P. Perona and A. Zisserman, 2003. Object class recognition by unsupervised scale-invariant learning. Proceedings of the 2003 IEEE Computer Society Conference on Computer Vision and Pattern Recognition Vol. 2, June 18-20, 2003, IEEE, Madison, Wisconsin, ISBN:0-7695-1900-8, pp: II-264-II-271.

Grabner, H., C. Leistner and H. Bischof, 2008. Semisupervised on-line boosting for robust tracking. Proceedings of the 10th European Conference on Computer Vision, October 12-18, 2008, Marseille, France, pp: 234-247.

Grabner, H., M. Grabner and H. Bischof, 2006. Real-time tracking via online boosting. BMVA, 1: 47-56.

Henriques, J.F., R. Caseiro, P. Martins and J. Batista, 2012. Exploiting the circulant structure of tracking-bydetection with kernels. Proceedings of the European Conference on Computer Vision, October 7-13, 2012, Springer, Berlin, Germany, ISBN:978-3-642-33764-2, pp: $702-715$.

Henriques, J.F., R. Caseiro, P. Martins and J. Batista, 2015. High-speed tracking with kernelized correlation filters. IEEE. Trans. Pattern Anal. Mach. Intell., 37: 583-596.

Hu, J., J. Lu and Y.P. Tan, 2016. Deep metric learning for visual tracking. IEEE. Trans. Circuits Syst. Video Technol., 26: 2056-2068.

Jia, X., H. Lu and M.H. Yang, 2012. Visual tracking via adaptive structural local sparse appearance model. Proceedings of the IEEE Conference on Computer Vision and Pattern Recognition, June 16-21, 2012, Rhode Island, USA., pp: 1822-1829.

Kwon, J. and K.M. Lee, 2010. Visual tracking decomposition. Proceedings of the Conference on Computer Vision and Pattern Recognition, June 13-18, 2010, San Francisco, CA., USA., pp: 1269-1276.

Li, G., B. Ma, J. Huang, Q. Huang and W. Zhang, $2016 \mathrm{~b}$. Beyond appearance model: Learning appearance variations for object tracking. Neurocomputing, 214 : 796-804.

Li, X., Q. Liu, Z. He, H. Wang and C. Zhang et al., 2016a. A multi-view model for visual tracking via. correlation filters. Knowl. Based Syst., 113: 88-99.
Liu, B., J. Huang, C. Kulikowski and L. Yang, 2013. Robust visual tracking using local sparse appearance model and K-selection. IEEE. Trans. Pattern Anal. Mach. Intell., 35: 2968-2981.

Liu, B., L. Yang, J. Huang, P. Meer and L. Gong et al., 2010. Robust and fast collaborative tracking with two stage sparse optimization. Proceedings of the European Conference on Computer Vision, September 5-11, 2010, Springer, Berlin, Germany, ISBN:978-3-64215560-4, pp: 624-637.

Liu, F., T. Zhou and J. Yang, 2016. Geometric affine transformation estimation via correlation filter for visual tracking. Neurocomputing, 214: 109120.

Mei, X. and H. Ling, 2009. Robust visual tracking using 11 minimization. Proceedings of the 2009 IEEE 12th International Conference on Computer Vision, September 29-October 2, 2009, IEEE, Kyoto, Japan, ISBN:978-1-4244-4420-5, pp: 1436-1443.

Mei, X., H. Ling, Y. Wu, E. Blasch and L. Bai, 2011. Minimum error bounded efficient \$/ell_1\$ tracker with occlusion detection. Proceedings of the 2011 IEEE Conference on Computer Vision and Pattern Recognition (CVPR'11), June 20-25, 2011, IEEE, Washington, USA., ISBN:978-1-4577-0394-2, Pages: 1257-1264.

Oron, S., A. Bar-Hillel, D. Levi and S. Avidan, 2015. Locally orderless tracking. Intl. J. Comput. Vision, 111: 213-228.

Patras, I. and E.R. Hancock, 2010. Coupled prediction classification for robust visual tracking. IEEE. Trans. Pattern Anal. Mach. Intell., 32: 1553-1567.

Qian, C. and Z. Xu, 2016. Robust visual tracking via sparse representation under subclass discriminant constraint. IEEE. Trans. Circuits Syst. Video Techn., 26: 1293-1307.

Ross, D.A., J. Lim, R.S. Lin and M.H. Yang, 2008. Incremental learning for robust visual tracking. Int. J. Comput. Vision, 7: 125-141.

Sabzmeydani, P. and G. Mori, 2007. Detecting pedestrians by learning shapelet features. Proceedings of the 2007 IEEE Conference on Computer Vision and Pattern Recognition (CVPR'07), June 17-22, 2007, IEEE, Minneapolis, Minnesota, ISBN:1-4244-1179-3, pp: 1-8.

Sevilla-Lara, L. and E. Learned-Miller, 2012. Distribution fields for tracking. Proceedings of the 2012 IEEE Conference on Computer Vision and Pattern Recognition (CVPR), June 16-21, 2012, IEEE, Providence, Rhode Island, ISBN:978-1 -4673-1226-4, pp: $1910-1917$. 
Shotton, J., J. Winn, C. Rother and A. Criminisi, 2009. Textonboost for image understanding: Multi-class object recognition and segmentation by jointly modeling texture, layout and context. Intl. J. Comput. Vision, 81: 2-23.

Tian, C., X. Gao, W. Wei and H. Zheng, 2015. Visual tracking based on the adaptive color attention tuned sparse generative object model. IEEE. Trans. Image Process., 24: 5236-5248.

Winn, J., A. Criminisi and T. Minka, 2005. Object categorization by learned universal visual dictionary. Proceedings of the 10th IEEE International Conference on Computer Vision (ICCV'05) Vol. 1, October 17-21, 2005, IEEE, Beijing, China, ISBN:07695-2334-X, pp: 1800-1807.

Wu, Y., J. Lim and M.H. Yang, 2013. Online object tracking: A benchmark. Proceedings of the 2013 IEEE Conference on Computer Vision and Pattern Recognition, June 23-28, 2013, IEEE, Portland, Oregon, USA., ISBN: 978-0-7695-4989-7, pp: 2411-2418.

Yang, F., H. Lu and M.H. Yang, 2014. Robust superpixel tracking. IEEE. Trans. Image Process., 23: 1639-1651

Yang, H., L. Shao, F. Zheng, L. Wang and Z. Song, 2011. Recent advances and trends in visual tracking: A review. Neurocomputing, 74: 3823-3831.
Yang, M., Y. Wu, M. Pei, B. Ma and Y. Jia, 2016. Online discriminative tracking with active example selection. IEEE. Trans. Circuits Syst. Video Technol., 26: 12791292.

Yuan, Y., J. Fang and Q. Wang, 2014. Robust superpixel tracking via depth fusion. IEEE. Trans. Circuits Syst. Video Technol., 24: 15-26.

Zhang, K. and H. Song, 2013. Real-time visual tracking via online weighted multiple instance learning. Pattern Recognit., 46: 397-411.

Zhang, K., L. Zhang and M.H. Yang, 2012. Real-time compressive tracking. Proceedings of the European Conference on Computer Vision, October 7-13, 2012, Springer, Berlin, Germany, ISBN:978-3-642-33711-6, pp: 864-877.

Zhang, L., D. Bi, Y. Zha, S. Gao and H. Wang et al., 2016. Robust and fast visual tracking via spatial kernel phase correlation filter. Neurocomputing, 204: 77-86.

Zhuang, B., H. Lu, Z. Xiao and D. Wang, 2014. Visual tracking via discriminative sparse similarity map. IEEE. Trans. Image Process., 23: 1872-1881.

Zhuang, B., L. Wang and H. Lu, 2016. Visual tracking via shallow and deep collaborative model. Neurocomputing, 218: 61-71. 